\title{
Experimental study on jet pulse assembly design and numerical simulation
}

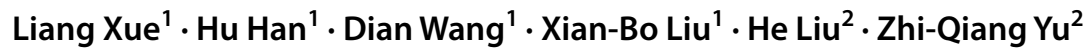

Received: 5 July 2019 / Published online: 29 November 2019

(c) The Author(s) 2019

\begin{abstract}
Jet pulse assembly is one of the main components of jet hydraulic oscillator. The pressure wave characteristics produced by jet pulse assembly have an important influence on the performance of the tool. In this paper, the structure and working principle of jet pulse assembly are studied, the mechanical analysis model of piston rod is established, the dynamic resistance ratio formula of jet pulse assembly is deduced, and the numerical simulation test of $89-\mathrm{mm}$ jet pulse assembly structure parameters is carried out. The results show that the piston rod downward stroke is driven by both the jet element driving force and the throttle plate load driving force, and can stably descend. The driving force of the piston rod upward stroke jet element is opposite to the load acting force of the throttle disc, and the jet driving force needs to be greater than the load resistance of the throttle disc to stably ascend. The dynamic resistance ratio formula is deduced. When the area of the end of the piston rod is reduced, the resistance of the throttle disc is reduced and the jet power is increased, thus solving the problem of insufficient power of the piston rod in the upstroke and ensuring the normal operation of the tool. Ten groups of numerical simulation tests were carried out, and it was found that the pressure amplitude and pressure drop of the tool decreased significantly with the increase of the tool size, and the error between the numerical simulation value and the theoretical calculation value was less than $9 \%$, which verified the correctness of the theory. It is suggested to select tools on site according to the drilling construction situation to ensure the drilling effect.
\end{abstract}

Keywords Friction reduction and torsion · Jet pulse assembly $\cdot$ Dynamic resistance ratio formula $\cdot$ Pressure amplitude $\cdot$ Numerical simulation

\section{Introduction}

With the gradual deepening of the development of oil fields, the number of complex structural wells such as high-angle wells, horizontal wells and multi-branch horizontal wells is increasing. The issue of realizing fast drilling and increasing the length of horizontal sections has become the focus of attention (Barton et al. 2011; Mccormick and Chiu 2011; Liu et al. 2016a, b). Since the 1980s, more and more downhole rotary drilling has been utilized in directional wells due to

Edited by Xiu-Qiu Peng

Liang Xue

xueliang200888@126.com

1 China University of Petroleum-Beijing, Beijing 102249, China

2 Bohai Drilling Engineering Technology Research Institute, Tianjin 300280, China many advantages, such as increasing the rate of mechanical drilling, increasing the footage of a single drill bit, and achieving well path control. However, the friction between the drill string and the borehole wall is large because of the large inclination angle of the well, which is resulting in low drilling rate (ROP), drilling tool wear and tear, and wellbore instability (Abdo and Al-Sharji 2014; Clausen et al. 2014; Martinez et al. 2013). In order to enhance the extending ability of directional wells and maximize the productivity of oil reservoirs, the problem of reducing friction torque has become the focus of research in the oil field. The most commonly used tools are axial oscillator tools (AOT) and lateral oscillator tools (LOT), which are used for reducing friction and reducing torque. When drilling, the tools are used together with the drill string or downhole drilling assembly to generate benign vibration and achieve the effect of reducing friction and resistance (Gee et al. 2015). The AOT typically uses a mud-driven sliding or rotary valve to generate a pressure pulse, and the pulse can be converted to 
an axial vibration motion by a vibrating nip, which can run on the AOT or transmitted to the AOT (Alali et al. 2012). The LOT typically uses an eccentric rotating mass driven by mud to create radial vibration between the wellbore and the tool, thereby reducing friction. In practical applications, both of them have effectively improved the drilling friction conditions, but the performance of AOT is significantly better than LOT (Zheng et al. 2003). The most widely used axial oscillator tools were developed by National Oilwell Varco (NOV) (Al-Buali et al. 2009). The tool was originally applied to continuous pipe drilling, and the application of axial oscillators in continuous tube drilling was introduced in the literature, respectively (Rasheed 2001; Maidla and Haci 2004; Al-Buali 2009). However, due to the good antifriction effect, the application range is extended to horizontal wells, high-angle wells, large displacement wells, etc. Since the successful development of the tool around 2000, it has been widely used in thousands of wells around the world. However, both AOT and LOT have slowed down the optimization and further application of these tools, which have complex structures such as deformable or movable parts and subsequent maintenance, technical difficulties associated with corrosion, wear and failure (Panchal et al. 2012).

By referring to the percussive and rotary drilling technology, Liu developed a fluidic axial oscillator tool (FAOT) (Liu 2014). The FAOT uses a bi-stable jet element as the core power component. Through the Coanda effect of the jet element, the high-pressure mud is controlled to flow into the upper and lower chambers of the cylinder, pushing the piston back and forth, and generating periodic pressure changes in the conical throttle disc. Liu He et al. used Computational Fluid Dynamics (CFD) simulation and experimental tests to verify the feasibility of the tool in directional drilling of horizontal wells (He et al. 2015). Besides, there are also many other fluidic oscillators that have been numerically investigated by using CFD simulations and have retained a high level of predictive accuracy (Bobusch et al. 2013; Tesai et al. 2013; Tesar and Smyk 2015; Pandey and Kim 2018). With no moving or deformable parts and all-metal construction, FAOT has the advantages of high-temperature resistance, corrosion-resistant environment, and other extreme environmental conditions, so its service life can extend significantly (Liu et al. 2016a, b). A large number of field tests have shown that compared with conventional drilling methods, the drilling efficiency is increased by more than $50 \%$ on average with the application of FAOT in directional well drilling. Besides, the tool can operate for more than $155 \mathrm{~h}$ in a complex underground environment without failure (CNPC 2016). Therefore, FAOT technology is a promising solution that for developing a new generation of complex well drilling process anti-friction tools.

However, it is found that there are some problems on FAOT affecting its working performance in the field application process: because the drilling fluid containing solidphase particles enters the FAOT, the high-speed jet will cause wear and erosion on the internal parts of the tool, and the jet element is most susceptible to erosion wear. The damage of the internal structure will directly lead to the abnormal operation of the tool and reduce the service life of the tool. FAOT will have unstable operation, large pressure consumption, small pressure amplitude, small axial vibration displacement, and poor drilling effect in actual operation (Zhang et al. 2017). The above problems show that the theoretical research on jet pulse assembly is not sufficient, especially the formulas for calculating structural parameters and hydraulic characteristics are lacking. Based on the mechanic analysis of the piston rod in the upstroke-downstroke, the dynamic resistance ratio formula of the piston rod movement is deduced for the $89-\mathrm{mm}$ jet pulse assembly. Besides, the structural parameters and hydraulic characteristics of the jet pulse assembly are quantitatively calculated, thus solving the problems existing in the design of the jet pulse assembly. The design theory has been verified by numerical simulation test, which is of great significance to the design and application of fluidic axial oscillator tool.

\section{Mechanical structure and principle}

As shown in Fig. 1, the jet pulse assembly with an outer diameter of $89 \mathrm{~mm}$ includes an outer structure and an inner structure (Mao et al. 2013). The external structure mainly consists of cylinder barrel and upper and lower joints. The internal structure consists of an upper gland, a jet element, a cylinder, an adjusting cone rod, a piston rod, a cylinder head, a spacer sleeve, and a throttle plate. The core components

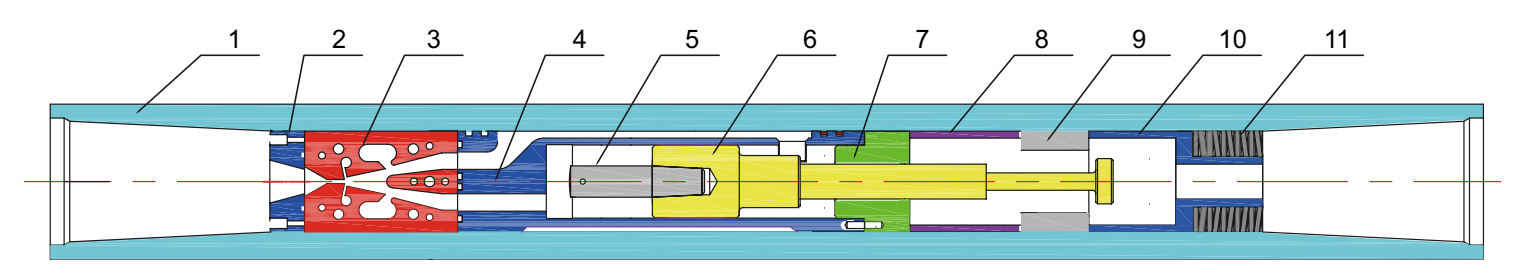

Fig. 1 Mechanical structure diagram of 89-mm jet-type pulse assembly. 1—outer cylinder, 2-upper gland, 3-jet element, 4-cylinder, 5adjusting cone rod, 6 — piston rod, 7— cylinder head, 8—-spacer sleeve, 9—-throttle plate, 10— disc spring cover, 11—diesel spring 
of jet pulse assembly are bi-stable jet element, piston rod, and throttle disc.

Bi-stable jet element has dual functions of power drive and commutation. The power driving principle is that during the movement of the upstroke-downstroke piston, the jet stabilizing wall acts on one side surface of the jet element. After the kinetic energy of the high-speed jet converted to high pressure, a pressure difference generate in the two output channels of the jet element. Then the pressure difference in the output channels communicate to the upper and lower chambers of the piston cylinder, which drive the piston rod to reciprocate up and down (Zhao et al. 2018).

The reversing principle is that when the piston in the piston cylinder moves to the upper and lower dead points, the jet flow of the jet element nozzle will switch. Then the jet flow will switch from one side of the attached wall flow to the other side. As a result, the high and low pressure distribution between the output channels and the upper and lower chambers of the piston cylinder will change, the pressure difference between the upper and lower end surfaces of the piston rod will also switch. And the piston rod in the piston cylinder will generate reverse movement under the action of reverse pressure difference, thus completing the movement reversing (Wang and Xue 2007, 2008).

The throttle plate is the component to generate pulse pressure. When the piston rod reciprocates inside the throttle plate, its flow area changes periodically, so the throttle pressure drop changes periodically. When the piston rod moves to the top dead centre, the piston rod is at the position where it has just entered the throttle plate. At this time, the overcurrent area is the smallest and the pressure drop generated is the largest. When the piston rod moves to the bottom dead centre, the piston rod reaches the disc spring sleeve. At this time, the overcurrent area is the largest and the pressure drop generated is the smallest. Under the action of periodic pressure, the jet pulse assembly generates a pressure wave, and the pressure wave acts on the vibration assembly. The vibration assembly generates axial vibration through a disc spring, thereby reducing friction and drag, preventing the bit from pressing and improving the penetration rate.

\section{Design theory}

The piston rod motion of jet pulse assembly includes upstroke and downstroke. The motion law and stress of the two strokes are completely different. The following is the mechanical analysis of the down and upstrokes.

\subsection{Mechanical analysis of downstroke}

As shown in Fig. 2, during the downstroke, the jet attaches to one side of the upper chamber, so the upper chamber is a high-pressure region, the low-pressure fluid in the lower chamber flows out of the evacuation channel, and the lower chamber is a low-pressure region. The piston rod moves downward under the jet pressure and the acting force at the throttle plate. The extraction of the pressure zone where the piston rod is located is simplified, and the theoretical analysis model of downstroke is established. As shown in Fig. 2, the area of the top end of the piston rod is $A_{1}$, wherein $A_{1}=\frac{\pi}{4} d_{1}^{2}$; the annular area of the middle section of the piston $\operatorname{rod}$ is $A_{2}$, wherein $A_{2}=\frac{\pi}{4}\left(d_{1}^{2}-d_{2}^{2}\right)$; the small diameter annular area of the piston $\operatorname{rod}$ is $A_{3}$, wherein $A_{3}=\frac{\pi}{4}\left(d_{2}^{2}-d_{3}^{2}\right)$; The end area of the piston $\operatorname{rod}$ is $A_{4}$, wherein $A_{4}=\frac{\pi}{4} d_{2}^{2}$.

During the downstroke, the piston rod is in four pressure zones. From left to right, the first is high-pressure zone $P_{1}$ (red zone) of the upper chamber, the second is the low-pressure zone $P_{2}$ (yellow zone) of the lower chamber. Then the third is the sub-low-pressure zone $P_{3}$ (green zone) of the spacer sleeve and the last is the lowest pressure zone $P_{4}$ (blue zone) of the disc spring sleeve, wherein $P_{1}>P_{2}>P_{3}>P_{4}$.

The positive direction is downward: the acting area of the upper chamber high pressure $P_{1}$ is $A_{1}$, and the acting direction of $P_{1} A_{1}$ is downward. The acting area of low pressure $P_{2}$ is $A_{2}$, and the acting direction of $P_{2} A_{2}$ is upward. The action area $A_{3}$ of sub-low pressure $P_{3}$ is upward, and the action direction of $P_{3} A_{3}$ is upward. The action areas of the lowest pressure $P_{4}$ are $A_{3}$ and $A_{4}$, respectively, the action direction of $P_{4} A_{3}$ is downward, and the action direction of $P_{4} A_{4}$ is upward. Therefore, the resultant force $F$ on the piston rod is:

$$
F=P_{1} A_{1}-P_{2} A_{2}-P_{3} A_{3}+P_{4} A_{3}-P_{4} A_{4}
$$

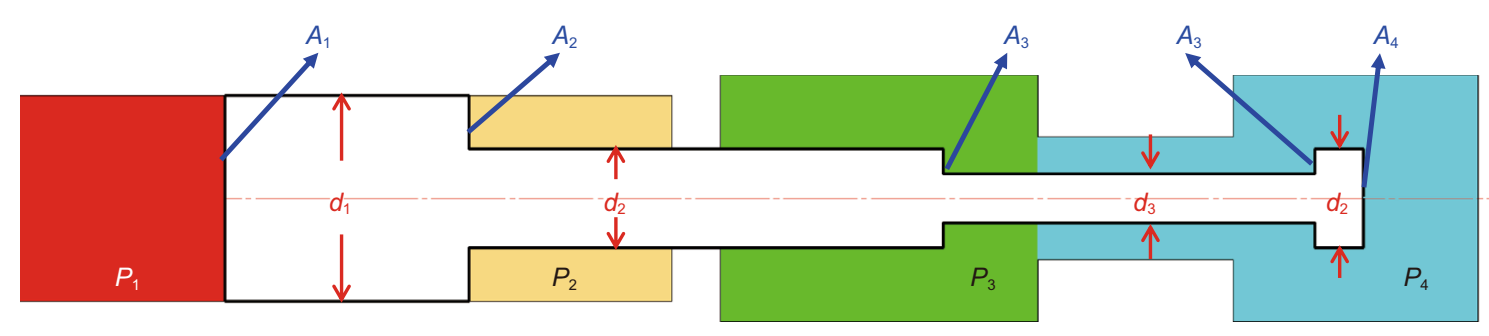

Fig. 2 Schematic diagram of downstroke mechanical analysis model 
As the fluid in the sub-low-pressure region $P_{3}$ passes through the throttle plate, the change of the flow area is small, so the generated flow pressure drop is small and can be ignored in calculation, so $P_{3} \approx P_{4}$, and $A_{1}=A_{2}+A_{4}$, which is arranged by substituting formula (1):

$F=\left(P_{1}-P_{2}\right) A_{2}+\left(P_{1}-P_{4}\right) A_{4}$

where $\left(P_{1}-P_{2}\right) A_{2}$ is defined as the jet power acting on the piston rod due to the high and low pressure difference generated by the jet wall, and $\left(P_{1}-P_{4}\right) A_{4}$ is defined as the throttle plate power generated by the diameter change of the piston rod at the throttle plate.

Therefore, through the mechanical analysis of the downstroke, it is concluded that the piston rod is affected by jet power and throttle plate power during the downstroke, so $F>0$, piston rod can move down smoothly, and the tool can work stably during the downstroke.

\subsection{Mechanical analysis of upstroke}

During the upstroke, the jet flow attaches to one side of the lower chamber during the upstroke, so the lower chamber is a high-pressure area, the low-pressure fluid in the upper chamber flows out of the evacuation passage, and the upper chamber is a low pressure. The piston rod moves upward under the jet flow pressure and the acting force at the throttle plate. As shown in Fig. 3, the pressure area where the piston rod is located is simplified and the upstroke mechanical analysis model is established.

During the upstroke, the piston rod is in five pressure zones. From left to right, the first is the low-pressure zone $P_{1}$ (yellow zone) of the upper chamber, and the second is the high-pressure zone $P_{2}$ (red zone) of the lower chamber. Then the third is the low-pressure zone $P_{3}$ (green zone) of the spacer sleeve, the fourth is the low-pressure zone $P_{4}$ (light blue zone) of the throttle plate, and the last is the lowest pressure zone $P_{5}$ (dark blue zone) of the disc spring sleeve, wherein $P_{2}>P_{1}>P_{3}>P_{4}>P_{5}$.

The positive direction is upward: the action area of low pressure $P_{1}$ is $A_{1}$, and the action direction of $P_{1} A_{1}$ is downward; The acting area of high pressure $P_{2}$ is $A_{2}$, and the acting direction of $P_{2} A_{2}$ is upward. The action area of low pressure $P_{3}$ is $A_{3}$, and the action direction of $P_{3} A_{3}$ is upward. The action area of low pressure $P_{4}$ is $A_{3}$, and the action direction of $P_{4} A_{3}$ is downward. The action area of the lowest pressure $P_{5}$ is $A_{4}$, and the action direction of $P_{5} A_{4}$ is upward. Therefore, the resultant force $F$ ' on the piston rod is

$F^{\prime}=-P_{1} A_{1}+P_{2} A_{2}+P_{3} A_{3}-P_{4} A_{3}+P_{5} A_{4}$

As the fluid in the sub-low-pressure region $P_{3}$ passes through the throttle plate, the change of the flow area is small, so the generated flow pressure drop is small and can be ignored in calculation, so $P_{3} \approx P_{4}$, and $A_{1}=A_{2}+A_{4}$, which is arranged by substituting formula (3):

$F^{\prime}=\left(P_{2}-P_{1}\right) A_{2}-\left(P_{1}-P_{5}\right) A_{4}$

In formula (4), $\left(P_{2}-P_{1}\right) A_{2}$ is defined as the jet power acting on the piston rod due to the high and low pressure difference generated by the jet wall, and $-\left(P_{1}-P_{5}\right) A_{4}$ is defined as the resistance at the throttle plate.

During the upstroke, the force on the piston rod includes jet power and resistance at the throttle plate. Whether it can move stably upward depends on the quantitative relationship between the two, which is the reason why the tool works unstably. The condition for stable upward stroke is that the jet power is not less than the throttle plate resistance, and the critical condition is that the jet power is equal to the throttle plate resistance.

Based on the mechanical analysis of up- and downstrokes, it is concluded that: firstly, during the downstroke, the piston rod moves downward and the throttle plate load is the power; during the upstroke, the piston rod moves upward, and the load of the throttle plate is resistance. Secondly, when the piston rod moves upward, the flow area of the throttle plate decreases continuously and the load resistance increases continuously. If the power of the tool is less than the resistance, the piston rod will be forced to reverse downward before reaching the top dead centre, affecting the normal work of the tool. Lastly, the condition for the piston rod to work stably upward is that the power

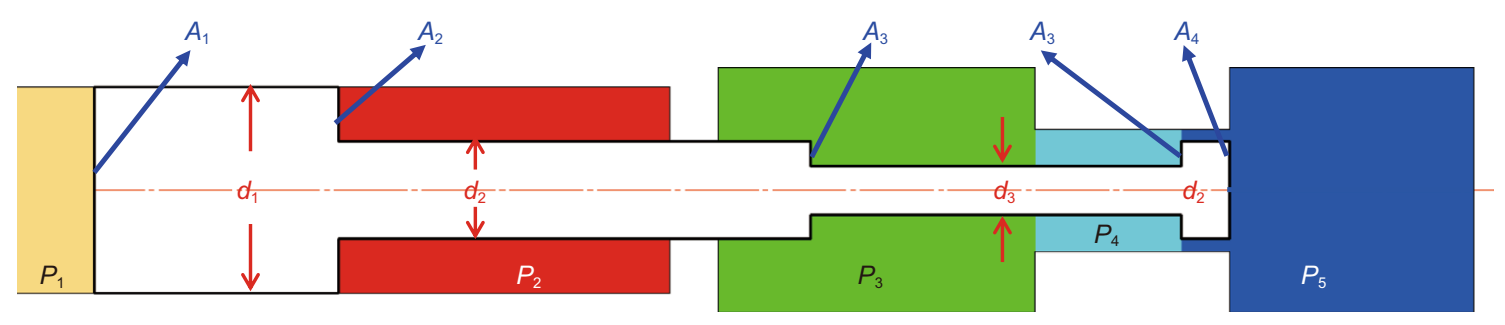

Fig. 3 Schematic diagram of pressure drop distribution (upstroke) 
is not less than the resistance and critical condition is that power equals resistance.

\subsection{Analysis of dynamic resistance ratio}

The condition for stable operation of the upstroke is that the power is not less than the resistance. The critical condition is that power equals resistance, so $F^{\prime}=0$, which is derived from Eq. (4):

$\left(P_{2}-P_{1}\right) A_{2}=C_{\mathrm{o}}\left(P_{1}-P_{5}\right) A_{4}$

$\frac{P_{2}-P_{1}}{P_{1}-P_{5}}=C_{\mathrm{o}} \frac{A_{4}}{A_{2}}$

where $C_{\mathrm{o}}$ represents the correction coefficient, $P_{1}$ represents the pressure of upper chamber, $P_{2}$ represents the pressure of lower chamber, and $P_{5}$ represents the pressure of disc spring sleeve. $A_{2}$ and $A_{4}$ represent the annular area of the middle section of the piston rod and the end area of the piston rod, respectively.

Since the overcurrent pressure drop from the spacer sleeve to the throttle plate and the friction pressure drop along the stroke are ignored in the mechanical analysis of the upstroke, a correction coefficient $C_{\mathrm{o}}$ is added to ensure the stable operation of the tool. When the correction coefficient is greater than 1, the power is greater than the resistance to ensure smooth upward movement of the piston rod.

From Eq. (6), the ratio of dynamic pressure difference to resistance pressure difference during the upstroke is equal to the ratio of the area of the end and middle end of the piston rod. When the jet velocity is constant, the dynamic pressure difference is also constant. At this time, when the area of the end of the piston rod reduces, the action area of the resistance pressure difference at the throttle plate will reduce. At the same time, as the area of the end reduces, the annular area of the middle end of the piston rod will increase, the area of the jet dynamic action will increase to increase the power. Thus, the problem of insufficient power of the piston rod on the upstroke is solved, and the tool can work normally.

\subsection{Analysis of pressure amplitude}

When the piston rod moves to the top dead centre, the end of the piston rod completely enters the throttle plate. The flow area of the throttle plate is the smallest, resulting in the largest flow pressure drop, so the minimum pressure here is P5. When the piston rod moves to the bottom dead centre, the flow area of the throttle plate is the largest and the generated flow pressure drop is the smallest, so the maximum pressure here is P4. So the flow pressure drop at the top dead centre is

$$
\Delta P_{1}=P_{3}-P_{5}=\frac{1}{2} \rho\left(C_{\mathrm{v}} \frac{Q}{A_{\mathrm{u}}}\right)^{2}=\frac{8 \rho C_{\mathrm{v}}^{2} Q^{2}}{\pi^{2}} \cdot \frac{1}{\left(d_{4}^{2}-d_{2}^{2}\right)^{2}}
$$

The flow pressure drop at the bottom dead centre is:

$\Delta P_{2}=P_{3}-P_{4}=\frac{1}{2} \rho\left(C_{\mathrm{v}} \frac{Q}{A_{\mathrm{b}}}\right)^{2}=\frac{8 \rho C_{\mathrm{v}}^{2} Q^{2}}{\pi^{2}} \cdot \frac{1}{\left(d_{4}^{2}-d_{3}^{2}\right)^{2}}$

where $P_{3}$ represents the pressure of the spacer sleeve, $P_{5}$ represents the minimum pressure at the throttle, and $P_{4}$ represents the maximum pressure at the disc spring sleeve. $\rho$ denotes drilling fluid density, $Q$ represents the drilling fluid displacement; $d_{2}, d_{3}$, and $d_{4}$ represent the piston rod end diameter, the smallest diameter of the piston rod, and throttle inner diameter, respectively. $A_{\mathrm{b}}$ represents the flow area of the throttle plate at the bottom dead centre. $A_{\mathrm{u}}$ represents that flow area of the throttle plate at the top dead centre. And $C_{\mathrm{v}}$ denotes velocity coefficient, its value is 0.75 here.

When the piston rod moves periodically in the throttle plate, the pressure amplitude is defined as $\Delta P_{\mathrm{w}}=P_{4}-P_{5}$. According to Eqs. (6)-(8), the difference between the two is the pressure amplitude after calculating the resistance pressure drop and the minimum overcurrent pressure drop. In addition, the tool pressure drop of field tools $\Delta P_{t}$ mainly consists of two parts: the jet pressure drop caused by highspeed jet at the nozzle and the pressure drop caused by pressure fluctuation at the throttle plate, ignoring the friction pressure drop along the path. In the theoretical calculation in this paper, the tool pressure drop is equal to the average value of the sum of the resistance pressure drop and the minimum overflow pressure drop plus the jet flow pressure drop of the nozzle.

According to the upstroke-downstroke mechanical analysis and pressure amplitude analysis, combined with the dynamic resistance ratio formula (6), a set of tool size and hydraulic parameters were calculated for the jet pulse assembly with an outer diameter of $89 \mathrm{~mm}$ and an inlet displacement of $10 \mathrm{~L} / \mathrm{s}$, while maintaining the jet flow velocity of $70 \mathrm{~m} / \mathrm{s}$. The parameter table is shown in Table 1 .

\section{Numerical simulation}

\subsection{Physical model}

In the numerical simulation, a jet pulse assembly with an outer diameter of $89 \mathrm{~mm}$ was selected, and a three-dimensional mechanical structure diagram was drawn according to its components and assembly drawings. Then a threedimensional calculation domain of flow field was generated according to the mechanical diagram, and finally a 
calculation grid was generated, thus establishing a numerical simulation physical model.

The jet pulse assembly has complicated mechanical structure and irregular structure of each component. The Solidworks software and splicing method were used to generate flow field calculation domain. As shown in Fig. 4, the flow field calculation domain includes the internal drainage basin of the jet element, the upper and lower cavity drainage basins of the cylinder body, the flow channels of the side wall, the spacer drainage basin, the throttle plate drainage basin, and the disc spring sleeve drainage basin (He et al. 2015). Fluid enters that jet element through the inlet, the jet element drives the piston rod to move up and down. The piston rod divides the cylinder body into an upper cavity and a lower cavity, the lower surface of the upper cavity and the upper surface of the lower cavity move up and down along with the piston rod. The reduce diameter at the lower end

Table 1 The parameters of tool dimensions and hydraulic parameters

\begin{tabular}{lllllllll}
\hline Displacement, $\mathrm{L} / \mathrm{s}$ & $d_{1}, \mathrm{~mm}$ & $d_{2}, \mathrm{~mm}$ & $d_{4}, \mathrm{~mm}$ & $d_{3}, \mathrm{~mm}$ & $\Delta P_{1}, \mathrm{MPa}$ & $\Delta P_{2}, \mathrm{MPa}$ & $\Delta P_{\mathrm{w}}, \mathrm{MPa}$ & $\Delta P_{\mathrm{t}}, \mathrm{MPa}$ \\
\hline \multirow{2}{*}{42} & 20 & 24.0 & 10 & 4.12 & 0.60 & 3.52 & 3.61 \\
& & 22 & 26.0 & & 3.52 & 0.40 & 3.11 & 3.21 \\
& 24 & 28.0 & & 3.03 & 0.28 & 2.75 & 2.91 \\
& & 26 & 30.5 & & 2.03 & 0.19 & 1.84 & 2.36 \\
& & 33.0 & & 1.41 & 0.13 & 1.28 \\
\hline
\end{tabular}
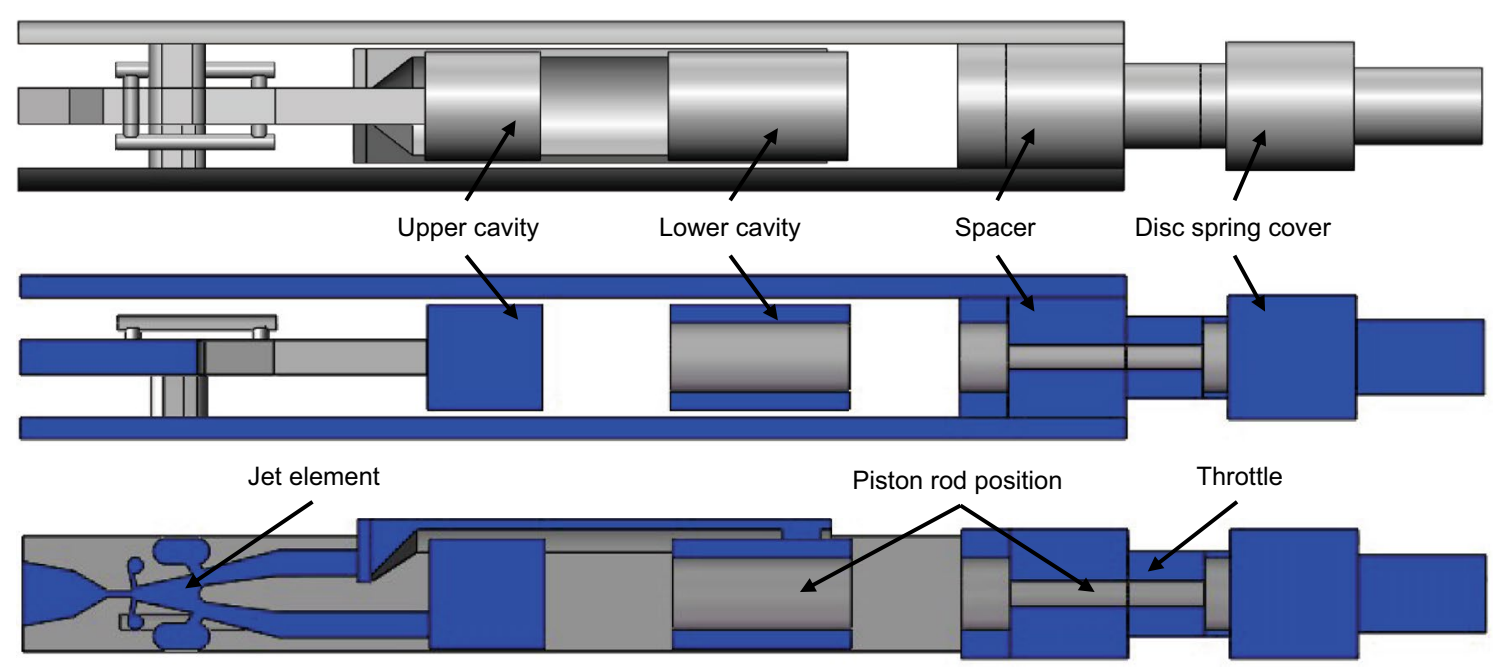

Fig. 4 Computational domain of jet pulsed assembly flow field

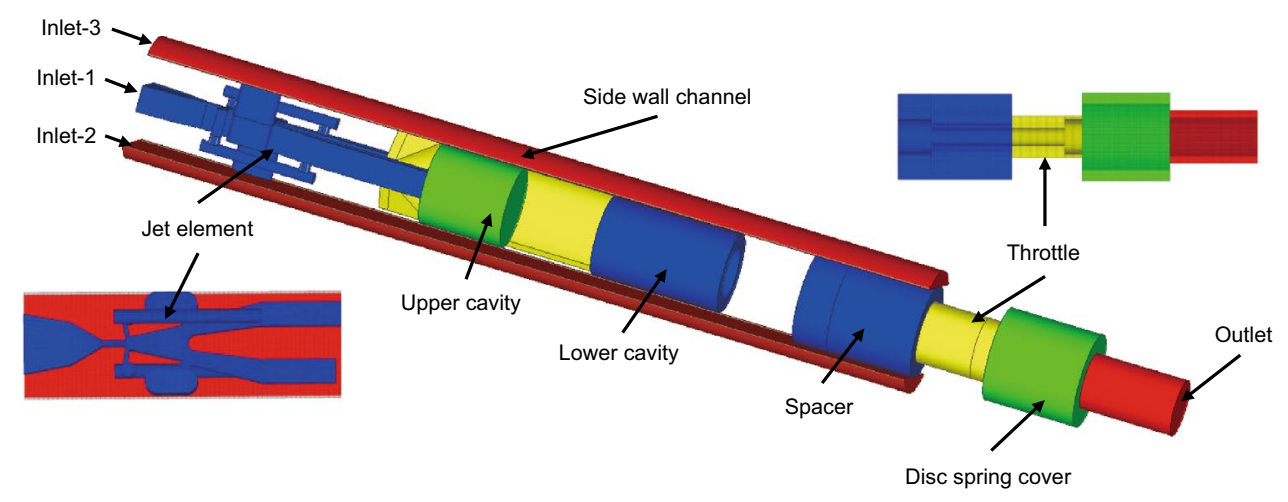

Fig. 5 CFD grid of 89-mm jet pulse assembly 
of the piston rod moves back and forth in the throttling disc and the disc spring sleeve, so that the overflow area changes.

The CFD grid of the jet pulse assembly is shown in Fig. 5. The mesh model has 1050,000 mesh cells, of which the volume mesh is 852,000, and the others are face mesh cells. The fluid domain consists of a volume mesh, which was used to define boundary conditions, initial conditions, walls, deformed meshes, and so on. Because the piston rod of $89-\mathrm{mm}$ jet pulse assembly has many diameter changes, grid division is carried out in the spacer sleeve and throttle plate basin, which is convenient for local grid encryption and capturing the hydraulic characteristics of fluid. This grid has three inlets, inlet- 1 was used to ensure the jet velocity is $70 \mathrm{~m} / \mathrm{s}$, while inlet- 2 and inlet- 3 were used to split flow, ensuring the total displacement of inlet is $10 \mathrm{~L} / \mathrm{s}$.

\subsection{Mathematical model}

After the physical model is established, based on the fluid mechanic's control equations and the discrete solution method, combined with the initial conditions and boundary conditions, the mathematical model of the $89-\mathrm{mm}$ jet pulsed assembly is established.

The fluid forms a high-speed jet in the jet element, and the flow state is very complicated, and there are physical processes such as vortex, wall attachment, and switching, which belong to turbulent flow. After previous experimental

Table 2 Parameters of structural dimensions of pulse assembly

\begin{tabular}{lllll}
\hline Case no. & $d_{1}, \mathrm{~mm}$ & $d_{2}, \mathrm{~mm}$ & $d_{3}, \mathrm{~mm}$ & $d_{4}, \mathrm{~mm}$ \\
\hline 1 & 42.0 & 20.0 & 10.0 & 24.0 \\
2 & 42.0 & 22.0 & 10.0 & 26.0 \\
3 & 42.0 & 24.0 & 10.0 & 28.0 \\
4 & 42.0 & 26.0 & 10.0 & 30.5 \\
5 & 42.0 & 28.0 & 10.0 & 33.0 \\
\hline
\end{tabular}

studies, the turbulence model has higher accuracy than other models for this system simulation (Wang 2004).

The numerical simulation of jet pulse assembly was mainly divided into the steady-state calculation of up- and downstrokes. Since the jet pulse assembly works at a given displacement, the three-inlet condition adopts the velocity inlet boundary condition. The jet velocity was kept at about $70 \mathrm{~m} / \mathrm{s}$ through the area ratio calculation, and the outlet pressure was defined as the natural outlet. The working pressure of inlet was set to zero, the other points are the pressure drop relative to the jet inlet. Besides, the object surface boundary adopts the non-slip boundary condition (Aram et al. 2017; Jeong and Kim 2018).

\subsection{Numerical simulation}

Five groups of tool structural dimensions were selected for numerical simulation tests. Each group of dimensions was divided into two groups of numerical experiments of upstroke-downstroke, totalling ten groups of experiments, with the sizes ranging from small to large in Table 2. When the displacement of the tool is $10 \mathrm{~L} / \mathrm{s}$, the working conditions of the pulse assembly with different sizes are simulated, and the hydraulic parameters calculated by the dynamic resistance ratio formula are compared with the numerical simulation test results.

The hydraulic characteristics of the pulse assembly at the top and bottom dead centres are numerically simulated. Since the numerical simulation are mainly aimed at analyzing the pressure amplitude and the pressure drop of the tool, the pressure contour of axial section is drawn, and the monitoring surface is selected on the pressure contour of up- and downstrokes to calculate the average pressure of the surface. As shown in Fig. 6, the pressure monitoring surface is divided into five parts.

As shown in Fig. 6, the pressure difference between Sects. 2 and 3 is the resistance pressure difference. The pressure difference between Sects. 4 and 5 is the minimum
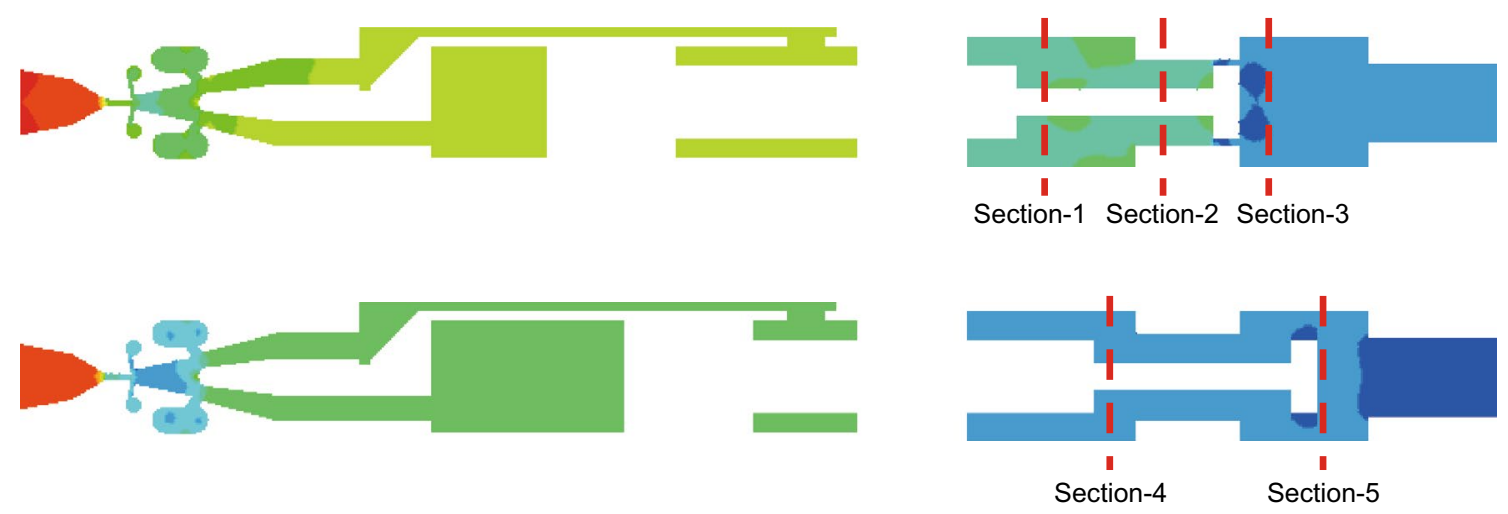

Fig. 6 Pressure monitoring surface of upstroke (upper) and pressure monitoring surface of downstroke (lower) 
overcurrent pressure difference. In addition, the difference between the resistance pressure difference and the minimum overcurrent pressure difference is the pressure amplitude value. However, the difference between Sects. 1 and 2 is used to characterize the overcurrent pressure difference between the spacer sleeve and the throttle plate ignored in the calculation of the formula. Change the parameters of different test models to carry out tests, record the pressure values at each section at different sizes, compare the pressure amplitude with the tool pressure drop, and analyze the errors.

\section{Results and discussion}

According to the sorted data, the numerical simulation hydraulic parameters in the up- and downstrokes of different test groups are shown in Table 3.

Figure 7 shows the pressure amplitude $\Delta P_{\mathrm{w}}$ obtained by formula calculation and numerical simulation and their error values for different case. It can be concluded that with the increase of the case number, that is, the flow area of the throttle plate increases, the pressure amplitude decreases significantly from 3.5 to $1.2 \mathrm{MPa}$, and the two are basically inversely proportional. When the overcurrent area increases, the piston rod moves to the place where the resistance is maximum (top dead centre), the increase of the overcurrent area significantly reduces the resistance pressure difference, while the minimum overcurrent pressure difference at the bottom dead centre decreases to a small extent, thus reducing the pressure amplitude. By calculating the relative error between the numerical simulation value and the formula calculation value, the error is less than $9 \%$, which meets the engineering calculation requirements. On the one hand, the relative error may be caused by the fact that the friction pressure drop along the path was ignored, the overcurrent pressure difference between the spacer and the throttle plate also be ignored. On the other hand, it is due to the calculation error caused by the discrete method in the numerical steadystate simulation. Therefore, in order to solve the problems of small pressure amplitude and weak axial vibration effect of the tool, it is necessary to design a small-sized piston rod

Table 3 Hydraulic parameters of upstroke-downstroke numerical simulation

\begin{tabular}{lllll}
\hline Case no. & $\Delta P_{1}, \mathrm{MPa}$ & $\Delta P_{2}, \mathrm{MPa}$ & $\Delta P_{\mathrm{w}}, \mathrm{MPa}$ & $\Delta P_{\mathrm{t}}, \mathrm{MPa}$ \\
\hline 1 & 0.59 & 4.08 & 3.48 & 3.59 \\
2 & 0.42 & 3.70 & 3.29 & 3.31 \\
3 & 0.25 & 3.04 & 2.79 & 2.89 \\
4 & 0.14 & 2.09 & 1.94 & 2.37 \\
5 & 0.11 & 1.52 & 1.41 & 2.07 \\
\hline
\end{tabular}

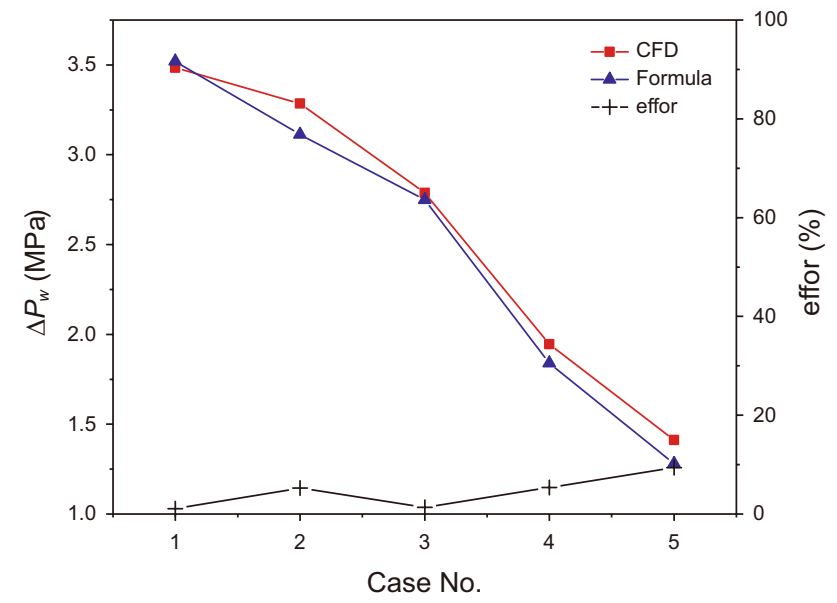

Fig. 7 Variation of pressure amplitude $\Delta P_{\mathrm{w}}$ with model size

and throttle disc to achieve the effects of reducing overcurrent resistance and increasing pressure amplitude.

Figure 8 shows the variation of tool pressure drop $\Delta P_{t}$ with the size of piston rod and throttle plate for different test groups. It can be found that the pressure drop of the tool decreases from 3.6 to 2.0 MPa with the increase of the size of the piston rod and throttle plate, and the result data obtained by formula is slightly different with that of the CFD simulation, but the error is very small and do not exceed $3 \%$. The fact is because when the size of the tool increases, the flow area at the throttle plate increases and the pressure drop caused by pressure fluctuation decreases, while the jet pressure drop remains unchanged, so the pressure drop of the tool decreases. Therefore, by designing a large-sized piston rod and throttle plate, the pressure drop of the tool can effectively reduce, thus solving the problem of large pressure drop of the tool at present.

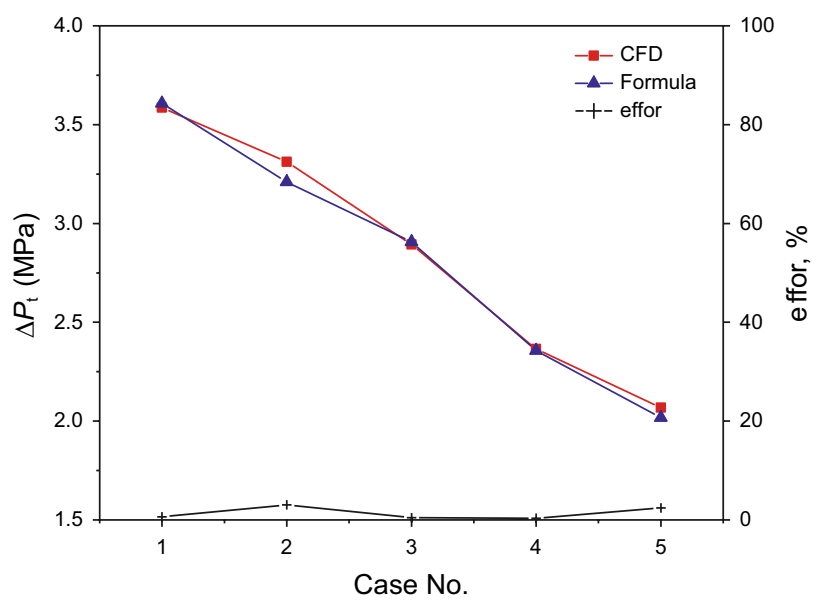

Fig. 8 Variation of tool pressure drop $\Delta P_{\mathrm{t}}$ with model size 
Table 4 Selection form of field tools (horizontal selection)

\begin{tabular}{|c|c|c|c|c|c|c|}
\hline Series & $d_{1}, \mathrm{~mm}$ & $d_{2}, \mathrm{~mm}$ & $d_{4}, \mathrm{~mm}$ & $d_{3}, \mathrm{~mm}$ & $\Delta P_{\mathrm{w}}, \mathrm{MPa}$ & $\Delta P_{\mathrm{t}}, \mathrm{MPa}$ \\
\hline 1 & 42 & 20 & 24.0 & 10 & 3.5 & 3.6 \\
\hline 2 & & 22 & 26.0 & & 3.1 & 3.2 \\
\hline 3 & & 24 & 28.0 & & 2.8 & 2.9 \\
\hline 4 & & 26 & 30.5 & & 1.8 & 2.4 \\
\hline 5 & & 28 & 33.0 & & 1.3 & 2.0 \\
\hline
\end{tabular}

According to the above analysis, large-sized tools can effectively reduce the pressure drop of tools, while smallsized tools can increase the pressure amplitude and improve the vibration effect. Therefore, the size of tools can be selected according to the needs of the site during site construction. When the ground pump is depressed, large-sized tools can be appropriately selected to reduce the pressure drop of tools. When the ROP is low, select a small-sized tool appropriately and increase the pressure amplitude, thus improving the ROP of the tool. The recommended selection table is shown in Table 4.

\section{Conclusion}

Based on the analysis of the structure and working principle of the jet-type pulse assembly, the mechanical analysis is carried out for the up- and downstroke, and five groups of jet pulse assembly with different parameters are selected for the up- and downstroke steady-state numerical simulation, and the following conclusions are obtained:

1. The piston rod can stably descend under the action of jet power and throttle plate load power during the downstroke of the jet pulse assembly. During the upstroke, both the jet power and the load resistance of the throttle plate are received, so the power must not be less than the resistance for stable work.

2. The formula of dynamic resistance ratio has been derived. The force and resistance of the piston rod during the upstroke are proportional to its acting area. While reducing the area of the piston rod end, the resistance can reduce and the jet power can be increase effectively.

3. The errors of numerical simulation and formula calculation are within the allowable range of engineering, which verifies the correctness of theoretical analysis. According to the numerical simulation, the pressure amplitude and the pressure drop of the tool decrease while the size of the tool increases, and the type selection of the tool should be carried out according to the actual use on site.
Open Access This article is distributed under the terms of the Creative Commons Attribution 4.0 International License (http://creativeco mmons.org/licenses/by/4.0/), which permits unrestricted use, distribution, and reproduction in any medium, provided you give appropriate credit to the original author(s) and the source, provide a link to the Creative Commons license, and indicate if changes were made.

\section{References}

Abdo J, Al-Sharji H. Investigation of inducing vibration to reduce friction of coiled tubing in deep drilling operations. In: Proceedings of the ASME international mechanical engineering congress and exposition, Montreal, QC, Canada 2014.

Alali A, Akubue VA, Barton SP, Gee R, Burnett TG. Agitation tools enables significant reduction in mechanical specific energy. In Proceedings of the SPE asia pacific oil and gas conference and exhibition. 2012. https://doi.org/10.2118/158240-ms.

Al-Buali MH, Dashash AA, Al-Shawly AS, Al-Guraini WK, Al-Driweesh SM, Bugrov V, Nicoll S. Maximizing coiled tubing reach during logging of extended horizontal wells using e-line agitator. In proceedings of the Kuwait international petroleum conference and exhibition, Kuwait City, Kuwait. 2009. https://doi. org/10.2118/127399-ms.

Aram S, Lee YT, Shan HC. Computational fluid dynamic analysis of fluidic actuator for active flow control applications. AIAA J. 2017;58(8):111-20. https://doi.org/10.1007/s00348-017-2392-0.

Barton SP, Baez F, Alali A. Drilling performance improvements in gas shale plays using a novel drilling agitator Device. In: Proceedings of the AADE national technical conference and exhibition, Houston, TX, USA. 2011. https://doi.org/10.2118/144416-ms.

Bobusch BC, Woszidlo R, Bergada JM, Nayeri CN, Paschereit CO. Experimental study of the internal flow structures inside a fluidic oscillator. Exp Fluids. 2013;54(6):1559. https://doi.org/10.1007/ s00348-013-1559-6.

Clausen JR, Schen AE, Forster I, Prill J, Gee R. Drilling with induced vibrations improves ROP and mitigates stick/slip in vertical and directional wells. In: Proceedings of the SPE drilling conference and exhibition, Fort Worth, TX, USA. 2014. https://doi. org/10.2118/168034-ms.

CNPC Bohai Drilling Engineering Company Limited. BH-HVT jetting hydraulic oscillator for drilling; Technical Report; CNPC Bohai Drilling Engineering Company Limited: Tianjin, China, 2016.

Gee R, Hanley C, Hussain R, Canuel L, Martinez J. Axial oscillation tools vs. lateral vibration tools for friction reduction - what's the best way to shake the pipe? Soc Pet Eng. 2015. https://doi. org/10.2118/173024-MS.

He JF, Yin K, Peng JM, Zhang XX, Liu H, Gan X. Design and feasibility analysis of a fluidic jet oscillator with application to horizontal directional well drilling. J Nat Gas Sci Eng. 2015;27:1723-31. https://doi.org/10.1016/j.jngse.2015.10.040. 
Jeong HS, Kim KY. Shape optimization of a feedback-channel fluidic oscillator. Eng Appl Comput Fluid Mech. 2018;12(1):169-81. https://doi.org/10.1080/19942060.2017.1379441.

Liu H. Theoretical analysis and experimental research on liquid jet oscillation tool. Ph.D. Thesis, Jilin University, Changchun, China. 2014. (Chinese).

Liu Y, Chen P, Wang X, Ma T. Modeling friction-reducing performance of an axial oscillation tool using dynamic friction model. J Nat Gas Sci Eng. 2016a;33:397-404. https://doi.org/10.1016/j. jngse.2016.05.034.

Liu H, Feng Q, Zhou J, Wu X, Zhao P, Wang J. Vibration frequency analysis of jetting hydraulic oscillator. China petroleum machinery. 2016b;44:20-4 (Chinese).

Maidla E, Haci M. Understanding torque: the key to slide-drilling directional wells. IADC/SPE drilling conference, Dallas. USA. 2004. https://doi.org/10.2118/87162-ms.

Mao Q, Hao PF, He F, Wang T, Wang JZ. Design and experimental study of fluidic micro-oscillator. J Exp Fluid Mech. 2013;112:79_ 82 (Chinese).

Martinez J, Carson CR, Canuel LAP, Burnett TG, Gee R. New technology enables rigs with limited pump pressure capacity to utilize the latest friction reduction technology. In: Proceedings of the SPE eastern regional meeting, Pittsburgh, PA, USA. 2013. https://doi. org/10.2118/165700-ms.

McCormick JE, Chiu TF. The practice and evolution of torque and drag reduction: theory and field results. In: Proceedings of the SPE annual technical conference and exhibition, Bangkok, Thailand. 2011. https://doi.org/10.2118/147100-ms.

Panchal N, Bayliss MT, Whidborne JF. Attitude control system for directional drilling bottom hole assemblies. IET Control Theory Appl. 2012;6(2):884-92. https://doi.org/10.1049/ iet-cta.2011.0438.
Pandey RJ, Kim KY. Numerical modeling of internal flow in a fluidic oscillator. J Mech Sci Technol. 2018;32(3):1041-8. https://doi. org/10.1007/s12206-018-0205-x.

Rasheed W. Extending the reach and capability of non-rotating BHAs by reducing axial friction. SPE/ICoTA coiled tubing roundtable, Houston. USA. 2001. https://doi.org/10.2118/68505-ms.

Tesai V, Zhong S, Rasheed F. New fluidic-oscillator concept for flowseparation control. AIAA J. 2013;51(2):397-405. https://doi. org/10.2514/1.J051791.

Tesar V, Smyk E. Fluidic low-frequency oscillator with vortex spin-up time delay. Chem Eng Process Process Intensif. 2015;90:6-15. https://doi.org/10.1016/j.cep.2015.02.001.

Wang FJ. Computational fluid dynamics analysis. Beijing: Tsinghua University Press; 2004. p. 39-43.

Wang ZM, Xue L. Study on the attached flow and alteration flow character in fluidic element of down hole pressure intensifier. J Hydrodyn. 2007;22(3):352-7 (Chinese).

Wang ZM, Xue L. Hydraulic parameter model for design of fluidics downhole boost compressor. Acta petrolei sinica. 2008;29(2):308 12 (Chinese).

Zhang XX, Peng JM, Liu H, et al. Performance analysis of a fluidic axial oscillation tool for friction reduction with the absence of a throttling plate. Appl Sci Basel. 2017;7(4):360. https://doi. org/10.3390/app7040360.

Zhao Y, Gong P, Liu ZG, Wang P, Feng D, Tu YL. Working principle and experimental research of a new type fluidic oscillator. Fluid Mach. 2018;83:13-7 (Chinese).

Zheng S, Jeffryes BP, Thomeer HV, Leising LJ. Method and apparatus to vibrate a downhole component. U.S. Patent 65718706. 2003. 